\section{nephron}

Practice
Nephron 2017; 136:298-301

DOI: $10.1159 / 000447673$
Received: January 28, 2016

Accepted after revision: June 15, 2016

Published online: July 8, 2016

\title{
Glomerular Filtration Rate Estimation in Renal and Non-Renal Solid Organ Transplantation
}

\author{
Mads Hornum Bo Feldt-Rasmussen \\ Department of Nephrology, Copenhagen University Hospital, Rigshospitalet, Copenhagen, Denmark
}

\section{Key Words}

Glomerular filtration rate estimation - Transplantation .

Equation performance

\begin{abstract}
Following transplantation (TX) of both renal and non-renal organs, a large proportion of patients have renal dysfunction. There are multiple causes for this. Chronic nephrotoxicity and high doses of calcineurin inhibitors are important factors. Preoperative and perioperative factors like hypertension, hypotension, drugs and infections may play a causative role as well. Organ-specific causes include hepatorenal syndrome, cirrhosis, low cardiac function, low respiratory function and diabetes developed both before and after TX. It is important to be able to perform precise and valid measurements or estimates of renal function in these patients, in order to accurately and safely dose immunosuppressive medication and perform and adjust the treatment and prophylaxis of renal dysfunction. This is a short overview and discussion of relevant studies and possible caveats of estimated glomerular filtration rate methods for use in renal and non-renal TX.

(c) 2016 S. Karger AG, Basel
\end{abstract}

Contribution from the CME course of the DIABESITY Working Group of the ERA-EDTA, Bergamo, December 4-5, 2015.
() 2016 S. Karger AG, Basel

\section{Introduction}

Serum creatinine is an unreliable marker of renal function in transplanted patients due to many factors including the catabolic state seen in chronic illness, malnutrition, the weight change induced by muscle wasting and fluid overload that is often present in these patients. Changes and fluctuations in the tubular secretion of creatinine is also an important factor [1]. Creatinine clearance measured by 24-hour collection of urine has been widely used, but is also biased by unstable tubular secretion of creatinine and the imprecise and cumbersome collections of urine.

Measured glomerular filtration rate (mGFR) with an exogenous filtration marker is the most accurate, either with inulin, ${ }^{51} \mathrm{CrEDTA}$-clearance, ${ }^{99 \mathrm{~m}} \mathrm{Tc}$ DTPA, iohexol or iothalamate. However, these methods are complex and more expensive to use in daily clinical routine [2].

Therefore many centers prefer to use the estimating methods, estimated GFR (eGFR) being an easy measure of GFR [3]. However, it should be clear that these methods are based on correlations or linear regression models between $\mathrm{P}$-creatinine, $\mathrm{P}$-cystatin $\mathrm{C}$ or both and variable patient variables and $\mathrm{mGFR}$, in rather heterogeneous populations. Despite high correlation quotients between eGFR and mGFR seen in many of these studies, it is ques-

Dr. Mads Hornum

Department of Nephrology

Copenhagen University Hospital

Rigshospitalet, Blegdamsvej 9, P 2131, DK-2100 Copenhagen (Denmark)

E-Mail mads.hornum@ regionh.dk 
tionable whether such correlations reflect a clinically useful agreement between eGFR and mGFR in different patient populations, which most often they do not. In this context, it is important to look at predictive performance of different equations, that is, absolute bias, relative bias and accuracy, as suggested in the KDOQI guidelines [4].

The absolute bias expresses the systematic deviation from the gold standard measurement of GFR and is given by the mean difference between eGFR and mGFR. The relative bias is expressed as the proportion of true GFR represented by the absolute bias. The distribution of the differences between estimated and true GFR accounts for the accuracy of the GFR estimates and can be assessed by the proportion of predicted GFR falling within 30 and $50 \%$ of the true GFR. However, there are typically wide ranges of agreement and this fact makes many equations too imprecise and not acceptable for clinical decision making.

We ask this question: are estimating methods applicable for use as a tool for correct measurement of renal function compared to direct methods in transplanted patients? The question still remains unanswered since no exact answers have so far been given.

\section{Kidney Transplantation}

The KDOQI guidelines classify chronic kidney disease (CKD) based on the presence of kidney damage and/or the impairment of kidney function. This underlines the need for a reliable method for the measurement of kidney function in various clinical conditions including kidney transplanted patients. This has been addressed in a number of studies.

First, in 154 kidney transplantation (TX) patients, Nankivell et al. [3] tested 6 published methods for the prediction of GFR from serum creatinine using ${ }^{99 \mathrm{~m}} \mathrm{Tc}$ DTPA clearance as a reference method. All methods overestimated GFR at lower levels. The relationship between serum creatinine and GFR was dependent on factors that alter muscle mass and muscle catabolic rate. Furthermore, clinical events such as acute tubular necrosis and chronic rejection influenced GFR. Two simplified formulas and one more detailed was derived from a database of 511 isotopic GFR measurements from kidney TX patients, including factors such as gender, height, body weight and height, serum urea, years on dialysis, numbers of rejections, infective episodes and prednisolone dose. These formulas were more ac-

GFR Estimation in TX curate, had the least overall error in predicting GFR compared to the published formulas and formula B had the best combination of simplicity and accuracy across a range of GFR.

Second, a prospective study in 284 kidney TX patients, performed by Mariat et al. [5] compared renal function measured by inulin clearance with eGFR by the Cockcroft-Gault (CG) and the Modification of Diet in Renal Disease (MDRD) 7 and abbreviated version equations and categorized them into the different stages of CKD according to KDOQI 2002 guidelines.

The equations overestimated GFR for CKD stages 3 and 4 and underestimated GFR for stage 2 . The bias increased as GFR declined to stage 5 for all equations, and the least biased equations were the MDRD formulas.

In the whole population, the MDRD equation were more accurate than the CG equation regarding performance and as for bias, accuracy tended to be lower with decreasing CKD. A number of reasons are listed to explain the poor performance, especially less muscle mass in transplanted patients. No information about nephron mass is available, and the choice and calibration of the assays used to determine the serum creatinine concentration is pinpointed as a potential misleading factor, and in general, the equations are developed in non-TX patients, which makes them less clinically relevant in transplanted patients.

Third, Luis-Lima et al. [6] evaluated the performance of 51 different formulas in predicting GFR in 193 renal transplant recipients and concluded that the formulas do not properly reflect renal function in kidney TX, which makes the use of the formulas in clinical practice unreliable. The authors also suggest using concordance correlation coefficient, total deviation index and coverage probability [7] when comparing the different formulas.

Fourth, in another study, Gaspari et al. [8] evaluated GFR decline and showed that eGFR does not reflect renal function decline over time.

In a systematic review, Harman et al. [9] demonstrated considerable heterogeneity in the performance of cystatin C-based estimating equations in the renal transplant population. Large between-study differences were presented, likely due to calibration issues with the measurement of cystatin $\mathrm{C}$ as well as differences in the study populations and GFR measurement techniques [9]. The Le Bricon equation was the most accurate cystatin C-based equation [10] and for a combined creatinine-cystatin $\mathrm{C}$ formula, it was the Stevens equation [11] that was most accurate.

Nephron 2017;136:298-301 


\section{Liver TX}

Estimation of renal function by serum creatinine is inadequate and inaccurate in cirrhotic patients, frequently overestimating GFR. In a study by Gonwa et al. [12], the performance of currently used GFR estimates by the CG equation, the Nankivell equation and the equations from the MDRD study $(6,5$ and 4 variables) were compared with $\mathrm{I}^{125}$ iothalamate $\mathrm{mGFR}$ pre and post TX. The MDRD 6 -variable equation was the most accurate; however, only at 1 and 5 years post TX, 67 and 64\%, respectively, of eGFR were within $30 \%$ of the mGFR [12].

The authors conclude that care should be taken when attempting to estimate GFR in liver transplanted patients. This has led to the development of cystatin C-derived formulas, since cystatin $\mathrm{C}$ is independent of muscle mass and hepatic biosynthesis, and Gerhardt et al. [13] found that best overall performance for GFR estimates was derived from the Hoek equation with respect to bias, precision and accuracy [14].

\section{Heart TX}

Heart failure patients have low renal plasma flow caused by low cardiac output leading to decreased renal function before TX. There are major problems with both early and long-term renal failure and this has a negative impact on long-term mortality [15].

Recently, Kolsrud et al. [16] published a large study of the relationship between eGFR estimated by CG, MDRD and CKD Epidemiology Collaboration formulas and mGFR performed by ${ }^{51}$ CrEDTA in 416 heart TX patients. The correlations between eGFR and mGFR were only moderate. The level of agreement between eGFR and mGFR was very low, and the percentage of patients with eGFR within $30 \%$ of mGFR rarely reached $75 \%$.

In conclusion, it is evident that mGFR and not eGFR should be used in clinical decision making in heart TX.

\section{Lung TX}

A progressive loss of renal function is common early and late after lung TX and the accuracy of creatinine and eGFR is questionable $[17,18]$. Estimation of renal dysfunction before lung TX is highly dependent of pulmonary diagnosis [17]. Creatinine-based slopes, although they correlate with GFR slopes after LTX, underestimate the rate of GFR decline. In a prospective study, Broekroelofs et al. [19] examined these rates and estimating equations of GFR decline and compared them with iothalamate clearance and recommended the Levey estimation (MDRD 7 estimation) [20] as the most sensitive method to detect small GFR losses [19]. Despite the highly relevant problem, only few studies exist that compares eGFR and mGFR studies of renal decline in lung transplanted patients.

The performance of eGFR methods are questioned, with various accuracy, and mGFR methods are recommended for correct clinical decision making.

\section{Conclusion}

In summary, estimation of GFR in solid organ TX is poorly validated and numerous sources of bias exist. The reasons why GFR estimation formulas fail both in short- and long-term studies compared with measured formulas are addressed and are mainly due to the differences in patient characteristics, change in weight, muscle mass and medication over time. The use of estimating methods for GFR determination are especially troublesome in clinical trials where correct assessment of GFR are crucial in determining correct dosing of medication and determination of a valid outcome. The use of mGFR is mandatory whenever GFR is the primary outcome of a study. Validation studies of estimating equations for use in solid organ TX with the use of patient characteristics, donor factors, drugs in use, rejection and diagnoses are needed, and until these equations are documented to be valid, direct GFR measurement are recommended.

\section{Acknowledgments}

M.H. participated in performance of the mini review, data analysis and writing of the paper. B.F.-R. participated in performance of the mini review, data analysis and writing of the paper.

\section{Funding Sources}

M.H. was supported by the Helen Bjørnow Foundation.

\section{Disclosure Statement}

The authors have nothing to declare. 


\section{References}

1 Berglund F, Killander J, Pompeius R: Effect of trimethoprim-sulfamethoxazole on the renal excretion of creatinine in man. J Urol 1975; 114:802-808.

2 Stevens LA, Coresh J, Greene T, Levey AS: Assessing kidney function - measured and estimated glomerular filtration rate. $\mathrm{N}$ Engl J Med 2006;354:2473-2483.

3 Nankivell BJ, Gruenewald SM, Allen RD, Chapman JR: Predicting glomerular filtration rate after kidney transplantation. Transplantation 1995;59:1683-1689.

4 National Kidney Foundation: K/DOQI clinical practice guidelines for chronic kidney disease: evaluation, classification, and stratification. Am J Kidney Dis 2002;39(2 suppl 1):S1S266.

5 Mariat C, Alamartine E, Afiani A, Thibaudin L, Laurent B, Berthoux P, et al: Predicting glomerular filtration rate in kidney transplantation: are the K/DOQI guidelines applicable? Am J Transplant 2005;5:2698-2703.

6 Luis-Lima S, Marrero-Miranda D, GonzálezRinne A, Torres A, González-Posada JM, Rodríguez A, et al: Estimated glomerular filtration rate in renal transplantation: the nephrologist in the mist. Transplantation 2015; 99:2625-2633.

7 Lin L, Hedayat AS, Sinha B et al: Statistical methods in assessing agreement: models, issues, and tools. J Am Stat Assoc 2002;97:257270

8 Gaspari F, Ferrari S, Stucchi N, Centemeri E, Carrara F, Pellegrino M, et al: Performance of different prediction equations for estimating renal function in kidney transplantation. Am J Transplant 2004;4:1826-1835.
9 Harman G, Akbari A, Hiremath S, White CA, Ramsay T, Kokolo MB, et al: Accuracy of cystatin C-based estimates of glomerular filtration rate in kidney transplant recipients: a systematic review. Nephrol Dial Transplant 2013;28:741-757.

10 Le Bricon T, Thervet E, Froissart M, Benlakehal M, Bousquet B, Legendre C, et al: Plasma cystatin $\mathrm{C}$ is superior to 24 - $\mathrm{h}$ creatinine clearance and plasma creatinine for estimation of glomerular filtration rate 3 months after kidney transplantation. Clin Chem 2000;46(8 pt 1):1206-1207.

11 Stevens LA, Coresh J, Schmid CH, Feldman HI, Froissart M, Kusek J, et al: Estimating GFR using serum cystatin $\mathrm{C}$ alone and in combination with serum creatinine: a pooled analysis of 3,418 individuals with CKD. Am J Kidney Dis 2008;51:395-406.

12 Gonwa TA, Jennings L, Mai ML, Stark PC, Levey AS, Klintmalm GB: Estimation of glomerular filtration rates before and after orthotopic liver transplantation: evaluation of current equations. Liver Transpl 2004;10:301309.

13 Gerhardt T, Pöge U, Stoffel-Wagner B, Ahrendt M, Wolff M, Spengler U, et al: Estimation of glomerular filtration rates after orthotopic liver transplantation: evaluation of cystatin C-based equations. Liver Transpl 2006;12:1667-1672.

14 Hoek FJ, Kemperman FA, Krediet RT: A comparison between cystatin C, plasma creatinine and the Cockcroft and Gault formula for the estimation of glomerular filtration rate. Nephrol Dial Transplant 2003;18:20242031.
15 Alam A, Badovinac K, Ivis F, Trpeski L, Cantarovich M: The outcome of heart transplant recipients following the development of endstage renal disease: analysis of the Canadian organ replacement register (CORR). Am J Transplant 2007;7:461-465.

16 Kolsrud O, Ricksten SE, Holmberg E, Felldin M, Karason K, Hammarsten O, et al: Measured and not estimated glomerular filtration rate should be used to assess renal function in heart transplant recipients. Nephrol Dial Transplant 2016;31:1182-1189.

17 Hornum M, Burton CM, Iversen M, Hovind P, Hilsted L, Feldt-Rasmussen B: Decline in 51Cr-labelled EDTA measured glomerular filtration rate following lung transplantation. Nephrol Dial Transplant 2007;22:36163622.

18 Hornum M, Iversen M, Steffensen I, Hovind P, Carlsen J, Andersen LW, et al: Rapid decline in 51Cr-EDTA measured renal function during the first weeks following lung transplantation. Am J Transplant 2009;9:14201426.

19 Broekroelofs J, Stegeman CA, Navis GJ, de Haan J, van der Bij W, de Boer WJ, et al: Creatinine-based estimation of rate of long term renal function loss in lung transplant recipients. Which method is preferable? J Heart Lung Transplant 2000;19:256-262.

20 Levey AS, Bosch JP, Lewis JB, Greene T, Rogers N, Roth D: A more accurate method to estimate glomerular filtration rate from serum creatinine: a new prediction equation. Modification of diet in renal disease study group. Ann Intern Med 1999;130:461470 . 